PROCEEDINGS OF THE

AMERICAN MATHEMATICAL SOCIETY

Volume 138, Number 4, April 2010, Pages 1491-1494

S 0002-9939(09)10172-7

Article electronically published on December 2, 2009

\title{
A FORMULA ON SCATTERING LENGTH OF POSITIVE SMOOTH MEASURES
}

\author{
MASAYOSHI TAKEDA
}

(Communicated by Richard C. Bradley)

\begin{abstract}
M. Kac studied the scattering length probabilistically and conjectured that its semi-classical limit equals the capacity of the support of the potential. This conjecture has been proved independently by Taylor, Takahashi, and Tamura. In this paper we give another simple proof by the random time-change argument for Dirichlet forms and extend the previous results to positive measure potentials.
\end{abstract}

\section{INTRODUCTION}

In 4 and [5, M. Kac and J. Luttinger studied the scattering length $\Gamma(V)$ of a positive $L^{1}$-function $V$ on the 3-dimensional Euclidean space $\mathbf{R}^{3}$. They gave a probabilistic expression of $\Gamma(V)$,

$$
\Gamma(V)=\lim _{t \rightarrow \infty} \frac{1}{t} \int_{\mathbf{R}^{3}}\left(1-\mathbf{E}_{x}^{W}\left(e^{-\int_{0}^{t} V\left(B_{s}\right) d s}\right)\right) d x,
$$

where $\left(\mathbf{P}_{x}^{W}, B_{t}\right)$ is the Brownian motion on $\mathbf{R}^{3}$, and proved in 7 that for a compact set $K \subset \mathbf{R}^{3}$ with Kac regularity, $\Gamma\left(\alpha 1_{K}\right)$ converges to the capacity of $K$ as $\alpha \rightarrow \infty$. Here $1_{K}$ is the indicator function of $K$. Moreover, M. Kac conjectured in 4 that for any positive $L^{1}$-function $V$ with compact support,

$$
\gamma_{V}:=\lim _{\alpha \rightarrow \infty} \Gamma(\alpha V)
$$

equals the capacity of the support of $V$. M. Taylor [10 probabilistically verified the conjecture and H. Tamura [9] proved it analytically. Y. Takahashi 8 gave a new probabilistic representation of $\Gamma(V)$ for more general symmetric Markov processes and proved that if $V$ is a positive continuous function with compact support, then the limit $\gamma_{V}$ exists and depends only on the set $\{x: V(x)>0\}$. Our aim is to extend these results and to give another simple proof of the conjecture. In fact, we will prove the following theorem by the time-change argument for Dirichlet forms.

Received by the editors March 11, 2009, and, in revised form, August 19, 2009.

2010 Mathematics Subject Classification. Primary 60J45, 60J55; Secondary 31C25.

Key words and phrases. Scattering length, symmetric Markov process, Dirichlet form, time change.

The author was supported in part by Grant-in-Aid for Scientific Research (No. 18340033 (B)), Japan Society for the Promotion of Science.

(C)2009 American Mathematical Society Reverts to public domain 28 years from publication 
Theorem 1.1. Let $\mu$ be a finite smooth measure with fine support $F^{\mu}$. Then

$$
\lim _{\alpha \rightarrow \infty} \Gamma(\alpha \mu)=\operatorname{Cap}\left(F^{\mu}\right) .
$$

Here Cap is the capacity.

\section{A FORMUla FOR SCATtERING LENGTH}

Let $X=\left(\Omega, \mathbf{P}_{x}, X_{t}, \theta_{t}, \zeta\right)$ be an $m$-symmetric Hunt process on a locally compact separable metric space $E$. Here $m$ is a positive Radon measure on $E, \theta_{t}$ is the shift operator satisfying $X_{s}\left(\theta_{t}\right)=X_{s+t}$ identically for $s, t \geq 0$, and $\zeta$ is the lifetime of $X$. Let $\mu$ be a positive smooth measure on $E$ and denote by $\left\{A_{t}^{\mu}\right\}_{t \geq 0}$ a positive continuous additive functional in the Revuz correspondence to $\mu$ (cf. [3, Theorem 5.1.3]). We define its right continuous inverse of $A_{t}^{\mu}$ by

$$
\tau_{t}(\omega)=\inf \left\{s>0: A_{s}^{\mu}(\omega)>t\right\}, \quad(\inf \emptyset:=\infty) .
$$

Let $F^{\mu}$ denote the fine support of $\left\{A_{t}^{\mu}\right\}_{t \geq 0}$ :

$$
F^{\mu}=\left\{x \in E: \mathbf{P}_{x}\left(\tau_{0}=0\right)=0\right\} .
$$

We assume that the measure $\mu$ is finite, $\mu(E)<\infty$.

In this paper we define the scattering length by

$$
\Gamma(\mu)=\int_{E} \mathbf{E}_{x}\left(e^{-A_{\zeta}^{\mu}}\right) \mu(d x) .
$$

In fact, if the Hunt process $X$ is conservative, that is, $\mathbf{P}_{x}(\zeta=\infty)=1$, then the scattering length $\Gamma(\mu)$ is also expressed as

$$
\Gamma(\mu)=\lim _{t \rightarrow \infty} \frac{1}{t} \int_{E}\left(1-\mathbf{E}_{x}\left(e^{-A_{t}^{\mu}}\right)\right) m(d x) .
$$

Indeed, let $\left\{p_{t}^{\mu}\right\}_{t \geq 0}$ be the Feynman-Kac semigroup,

$$
p_{t}^{\mu} f(x)=\mathbf{E}_{x}\left(e^{-A_{t}^{\mu}} f\left(X_{t}\right)\right)
$$

for a bounded Borel function $f$. Denote by $\mathbf{P}_{x}^{\mu}$ the subprocess of $X$ by $e^{-A_{t}^{\mu}}$, $\mathbf{P}_{x}^{\mu}(d \omega)=e^{-A_{t}^{\mu}(\omega)} \cdot \mathbf{P}_{x}(d \omega)$. We see from [2, Theorem 2.2.2] that the Revuz measure of $A_{t}^{\mu}$ with respect to the subprocess $\mathbf{P}_{x}^{\mu}$ is also $\mu$ and from [6. (62.13)] that

$$
\mathbf{E}_{x}\left(\int_{0}^{t} e^{-A_{s}^{\mu}} d A_{s}^{\mu}\right)=\mathbf{E}_{x}^{\mu}\left(A_{t}^{\mu}\right) .
$$

Hence by [3, Theorem 5.1.3 (iii)],

$$
\begin{aligned}
\left\langle m, 1-\mathbf{E}_{x}\left(e^{-A_{t}^{\mu}}\right)\right\rangle & =\left\langle m, \mathbf{E}_{x}\left(\int_{0}^{t} e^{-A_{s}^{\mu}} d A_{s}^{\mu}\right)\right\rangle=\left\langle m, \mathbf{E}_{x}^{\mu}\left(A_{t}^{\mu}\right)\right\rangle \\
& =\int_{0}^{t}\left\langle\mu, p_{s}^{\mu} 1\right\rangle d s .
\end{aligned}
$$

Here we use the notation $\langle\nu, f\rangle=\int_{E} f(x) \nu(d x)$ for a measure $\nu$ and a function $f$. Since $p_{t}^{\mu} 1(x)$ converges to $\mathbf{E}_{x}\left(e^{-A_{\infty}^{\mu}}\right)$ as $t \rightarrow \infty$, we have the equation (2.2).

Let Cap be the 0-capacity associated with the Dirichlet form $(\mathcal{E}, \mathcal{D}(\mathcal{E}))$ of $X$ (Chapter 2 in [3]). Then the next lemma leads us to Theorem 1.1. 


\section{Lemma 2.1.}

$$
\Gamma(\alpha \mu)=\alpha \int_{E} \mathbf{E}_{x}\left(e^{-\alpha A_{\zeta}^{\mu}}\right) \mu(d x) \uparrow \operatorname{Cap}\left(F^{\mu}\right), \quad \alpha \uparrow \infty .
$$

For the proof of Lemma 2.1, we apply the time-change theory for Dirichlet forms ([1], 3]).

\section{Proof of Lemma 2.1}

We define the time-changed process by $Y_{t}=X_{\tau_{t}}$. Then $Y_{t}$ is a $\mu$-symmetric Markov process with state space $F^{\mu}$ and life time $A_{\zeta}^{\mu}$ (cf. [3, Theorem 6.2.1], [6, Theorem 65.9]). Denote by $\check{\mathbf{P}}_{x}$ the law of the time-changed process $Y_{t}$ and by $\check{\zeta}$ its life time. We then have for $x \in F^{\mu}$ :

$$
\begin{aligned}
\mathbf{E}_{x}\left(e^{-\alpha A_{\zeta}^{\mu}}\right) & =\check{\mathbf{E}}_{x}\left(e^{-\alpha \check{\zeta}}\right)=1-\alpha \check{\mathbf{E}}_{x}\left(\int_{0}^{\check{\zeta}} e^{-\alpha t} d t\right) \\
& =1-\alpha \check{R}_{\alpha} 1(x),
\end{aligned}
$$

where $\check{R}_{\alpha}$ is the $\alpha$-resolvent of $Y_{t}$ and 1 is the identity function on $F^{\mu}: 1=1_{F^{\mu}}(x)$. Hence the left-hand side of (2.3) equals $\alpha\left(1,1-\alpha \check{R}_{\alpha} 1\right)_{\mu}$.

Let $(\breve{\mathcal{E}}, \mathcal{D}(\breve{\mathcal{E}}))$ be the Dirichlet form on $L^{2}\left(F^{\mu} ; \mu\right)$ generated by the time-changed process $Y_{t}$. Noting that the function $1_{F^{\mu}}$ is in $L^{2}\left(F^{\mu} ; \mu\right)$ by the finiteness of $\mu$, we know that if $1_{F^{\mu}} \in \mathcal{D}(\check{\mathcal{E}})$, then $\check{\mathcal{E}}^{(\alpha)}(1,1):=\alpha\left(1,1-\alpha \check{R}_{\alpha} 1\right)_{\mu}$ is non-decreasingly convergent to $\check{\mathcal{E}}(1,1)$ as $\alpha \uparrow \infty$ (in [3, the form $\check{\mathcal{E}}^{(\alpha)}$ is said to be the approximating form of $\check{\mathcal{E}})$. The Dirichlet space $\mathcal{D}(\overline{\mathcal{E}})$ is identified by Theorem 6.2.1 in [3], and the Dirichlet form $\check{\mathcal{E}}$ is expressed by the original Dirichlet form $\mathcal{E}$ ([3, Theorem 6.2.1]); in particular,

$$
\check{\mathcal{E}}(1,1)=\mathcal{E}\left(H_{F^{\mu}} 1, H_{F^{\mu}} 1\right), \quad H_{F^{\mu}} 1(x)=\mathbf{E}_{x}\left(1_{F^{\mu}}\left(X_{\sigma_{F^{\mu}}}\right) ; \sigma_{F^{\mu}}<\infty\right),
$$

where $\sigma_{F^{\mu}}=\inf \left\{t>0: X_{t} \in F^{\mu}\right\}$. We thus have

$$
\Gamma(\alpha \mu)=\alpha\left(1,1-\alpha \check{R}_{\alpha} 1\right)_{\mu} \uparrow \check{\mathcal{E}}(1,1)=\mathcal{E}\left(H_{F^{\mu}} 1, H_{F^{\mu}} 1\right)
$$

as $\alpha \uparrow \infty$. Since $F^{\mu}$ is a nearly Borel, finely closed set (cf. [3. p. 192]), $\mathbf{P}_{x}\left(X_{\sigma_{F} \mu} \in\right.$ $\left.F^{\mu}\right)=1$ and thus

$$
H_{F^{\mu}} 1(x)=\mathbf{P}_{x}\left(\sigma_{F^{\mu}}<\infty\right) .
$$

Therefore, the right-hand side of (3.1) equals $\operatorname{Cap}\left(F^{\mu}\right)$ by [3, Theorem 4.3.3].

If $1_{F^{\mu}} \notin \mathcal{D}(\check{\mathcal{E}})$, then $\lim _{\alpha \rightarrow \infty} \check{\mathcal{E}}^{(\alpha)}(1,1) \uparrow \infty$ as $\alpha \uparrow \infty$ and $\operatorname{Cap}\left(F^{\mu}\right)=\infty$. The proof of the lemma is complete.

Finally we would like to make a comment on the fine support $F^{\mu}$. Let $F$ be the topological support of $\mu$. Then the set $F^{\mu} \backslash F$ is of zero capacity, while $F \backslash F^{\mu}$ is not necessarily of zero capacity (cf. [3, §5.1]). We see that

$$
\mathbf{P}_{x}\left(A_{\zeta}^{\mu}>0\right)=\mathbf{P}_{x}\left(\sigma_{F^{\mu}}<\zeta\right)
$$

Indeed, noting that if $\sigma_{F^{\mu}}<\zeta$, then $A_{\zeta}^{\mu}=A_{\zeta}^{\mu}\left(\theta_{\sigma_{F}^{\mu}}\right)$; and if $\sigma_{F^{\mu}}=\infty$, then $A_{\zeta}^{\mu}=0$, we have

$$
\begin{aligned}
\mathbf{P}_{x}\left(A_{\zeta}^{\mu}>0\right) & =\mathbf{P}_{x}\left(A_{\zeta}^{\mu}>0, \sigma_{F^{\mu}}<\zeta\right)+\mathbf{P}_{x}\left(A_{\zeta}^{\mu}>0, \sigma_{F^{\mu}}=\infty\right) \\
& =\mathbf{P}_{x}\left(A_{\zeta}^{\mu}\left(\theta_{\sigma_{F}}\right)>0, \sigma_{F^{\mu}}<\zeta\right) \\
& =\mathbf{E}_{x}\left(\mathbf{P}_{X_{\sigma_{F}}}\left(A_{\zeta}^{\mu}>0\right) ; \sigma_{F^{\mu}}<\zeta\right) \text { (by the strong Markov property). }
\end{aligned}
$$


Since $\mathbf{P}_{X_{\sigma_{F}}}\left(A_{\zeta}^{\mu}>0\right)=1$ on $\left\{\sigma_{F^{\mu}}<\zeta\right\}$, the right-hand side above equals $\mathbf{P}_{x}\left(\sigma_{F^{\mu}}<\zeta\right)$.

We consider the Brownian motion $\mathbf{P}_{x}^{W}$ on $\mathbf{R}^{3}$ and $\mu(d x)=1_{K}(x) d x$ with a compact set $K \subset \mathbf{R}^{3}$. Then the Kac regularity of $K$ is, in [7, defined by $\operatorname{Cap}\left(K \backslash F^{\mu}\right)=0$ and thus $\mathbf{P}_{x}^{W}\left(\sigma_{K}=\sigma_{F^{\mu}}\right)=1$. As a result, we see that if a compact set $K$ is $\operatorname{Kac}$ regular, then $\operatorname{Cap}\left(F^{\mu}\right)=\operatorname{Cap}(K)$.

\section{REFERENCES}

1. Z.-Q. Chen and M. Fukushima, Symmetric Markov Processes, Time Change and Boundary Theory, book manuscript (2009).

2. P.J. Fitzsimmons and R.K. Getoor, Revus measures and time changes, Math. Zeit. 199 (1988), 233-256. MR $958650(89 \mathrm{~h}: 60124)$

3. M. Fukushima, Y. Oshima and M. Takeda, Dirichlet Forms and Symmetric Markov Processes, De Gruyter (1994). MR:1303354 (96f:60126)

4. M. Kac, Probabilistic methods in some problems of scattering theory, Rocky Mountain J. Math. 4 (1974), 511-537. MR0510113 (58:23170)

5. M. Kac and J.-M. Luttinger, Scattering length and capacity, Ann. Inst. Fourier (Grenoble) 25 (1975), 317-321. MR0402079 (53:5902)

6. M. Sharpe, General theory of Markov processes, Pure and Applied Mathematics, 133, Academic Press (1988). MR958914 (89m:60169)

7. D.W. Stroock, The Kac approach to potential theory. I, J. Math. Mech. 16 (1967), 829-852. MR0208690 (34:8499)

8. Y. Takahashi, An integral representation on the path space for scattering length, Osaka J. Math. 7 (1990), 373-379. MR1066632 (91j:35083)

9. H. Tamura, Semi-classical limit of scattering length, Lett. Math. Phys. 24 (1992), 205-209. MR.1166749 (93m:81030)

10. M.E. Taylor, Scattering length and perturbations of $-\Delta$ by positive potentials, J. Math. Anal. Appl. 53 (1976), 291-312. MR.0477504 (57:17028)

Mathematical Institute, Tohoku University, Aoba, Sendai, 980-8578, Japan

E-mail address: takeda@math.tohoku.ac.jp 